\title{
THE LEAST SQUARE AND THE WEIGHTED LEAST SQUARE METHODS FOR ESTIMATING THE WEIBULL DISTRIBUTION PARAMETERS - A COMPARATIVE STUDY
}

In this paper we study the performance of the least square method and the weighted least square method for estimating the Weibull distribution parameters. In engineering practice these methods are commonly used due to their simplicity. The estimates of the parameters can be calculated easily by the closed-form formula. We consider three estimators of the cumulative distribution function and the weight factor proposed by Bergman (1986). The methods are compared in terms of the root mean square error and sample size. The comparison is based on the Monte Carlo simulation. The comparison shows that the weight factor improves the accuracy of the estimation the Weibull distribution parameters.

Keywords: Weibull distribution, parameter estimation, least square method, weighted least square method, root mean square error.

\section{Introduction}

The Weibull distribution is one of the widely used distributions in engineering practice. It is named after Walodi Weibull (18871979), who popularized its use in the theory of reliability, especially for metallurgical failure models. Moreover, the Weibull distribution is useful for description of the life time of the machine components, for description of mechanical properties of the materials as fatigue of materials and strength of materials.

We consider the two parameter Weibull distribution. The probability density function of the Weibull distribution with parameters $c>0$ and $\delta>0$, abbreviated $W(c, \delta)$, is given by

$$
f(x)=\frac{c}{\delta^{c}} x^{c-1} \exp \left(-\left(\frac{x}{\delta}\right)^{c}\right)
$$

where $x>0, c$ is the shape parameter and $\delta$ is the scale parameter.

The cumulative distribution function of the Weibull distribution is

$$
F(x)=1-\exp \left(-\left(\frac{x}{\delta}\right)^{c}\right), x>0 .
$$

The mean $\mu$ and the variance $\sigma^{2}$ of the Weibull distribution are

$$
\begin{aligned}
& \mu=\delta \Gamma\left(1+\frac{1}{c}\right), \\
& \sigma^{2}=\delta^{2}\left[\Gamma\left(1+\frac{2}{c}\right)-\Gamma^{2}\left(1+\frac{2}{c}\right)\right],
\end{aligned}
$$

where $\Gamma(a)$ is the gamma function defined by $\Gamma(a)=\int_{0}^{\infty} x^{a-1} e^{-x} d x$, $a>0$.

\footnotetext{
* Ivana Pobocikova, Zuzana Sedliackova
}

Department of Applied Mathematics, Faculty of Mechanical Engineering, University of Zilina, Slovakia, E-mail: ivana.pobocikova@fstroj.uniza.sk
The failure rate function of the Weibull distribution is given by

$$
H(x)=\frac{c}{\delta^{c}} x^{c-1} .
$$

Fig. 1 shows the effect of the shape parameter $c$ on the density function for different values $c$ and $\delta=1$. Fig. 2. shows the effect of the scale parameter $\delta$ on the density function for different values $\delta$ and $c=2$.

In this paper we study the performance of the methods for estimating the Weibull distribution parameters $c$ and $\delta$. The estimates of the parameters $c$ and $\delta$ can be obtained in more ways. The commonly used methods are the maximum likelihood method (MLM), the method of moments (MOM), the least square method (LSM) and the weighted least square method (WLSM). The MLM is the most popular for its efficiency and good properties, but the calculation is complicated. The estimates of the parameters can be obtained only numerically. Several authors have studied and compared performance of the methods for estimating the Weibull distribution parameters, e. g. Bergman [1], Chu and Ke [2], Faucher and Tyson [3], Lu, Chen and $\mathrm{Wu}$ [4], Trustrum and Jayatilaka [5], Wu, Zhou and Li [6], Zerda [7].

Here, we consider the least square method (LSM) and the weighted least square method (WLSM), each with three estimators of the cumulative distribution function $F(x)$. In engineering practice these methods are commonly used due to their simplicity. The estimates of the parameters can be calculated easily by the closedform formula. The methods are compared using the Monte Carlo simulation. The comparison is based on the root mean square error (RMSE) and the sample size $n$. Based on the simulation study we recommend the methods which have better performance. 


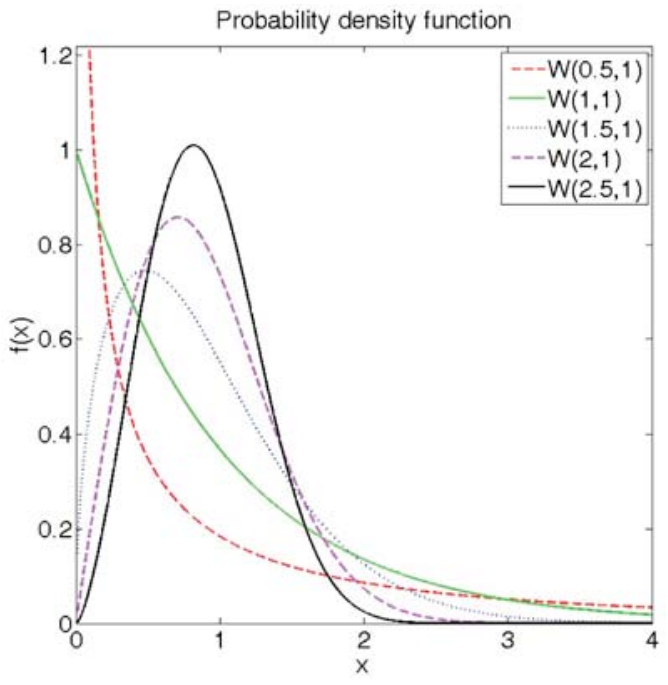

Fig. 1 Shows the effect of the shape parameter $c$ on the density function for different values $c$ and $\delta=1$

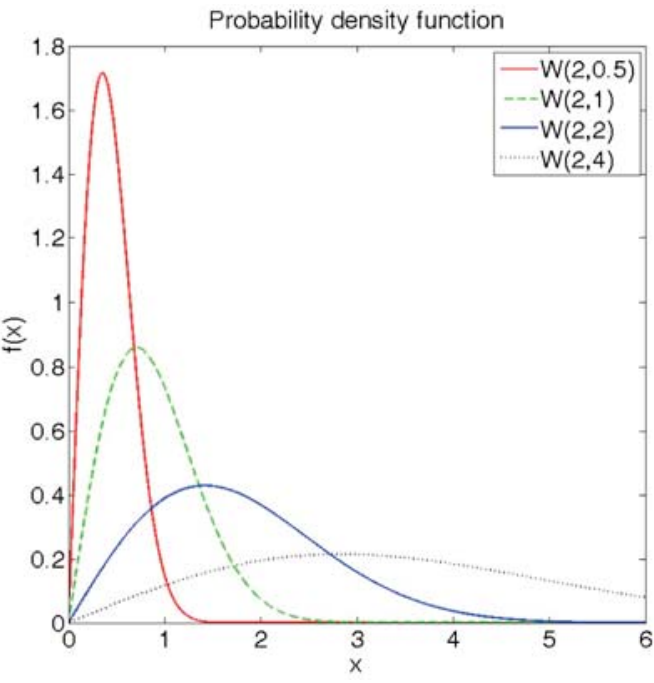

Fig. 2 Shows the effect of the scale parameter $\delta$ on the density function for different values $\delta$ and $c=2$

\section{Estimation of the parameters of the Weibull distribution}

In this section we introduce the methods for estimating the Weibull distribution parameters. The estimates of the parameters $c$ and $\delta$ denote $\hat{c}$ and $\hat{\delta}$, respectively. Let $X_{1}, X_{2}, \ldots, X_{n}$ be a random sample of size $n$ from the Weibull distribution $W(c, \delta)$ and let $x_{1}$, $x_{2}, \ldots, x_{n}$ be a realization of a random sample.

\section{Least square method}

Now, the cumulative distribution function (1) will be transformed to a linear function. From (1) by two logarithmic calculations we obtain

$$
\ln [-\ln (1-F(x))]=c \ln x-c \ln \delta
$$

Let $Y=\ln [-\ln (1-F(x))], X=c \ln x, \beta_{1}=c$ and $\beta_{0}=$ $=-c \ln \delta$. Then the equation (2) can be written as

$$
Y=\beta_{1} X+\beta_{0}
$$

Now let $X_{(1)}, X_{(2)}, \ldots, X_{(n)}$ be the order statistics of $X_{1}, X_{2}, \ldots$, $X_{n}$ and let $x_{(1)}<x_{(2)}<\ldots<x_{(n)}$ be observed ordered observations. To estimate the values of the cumulative distribution function $F(x)$ we can use the folloving methods

$$
\begin{aligned}
& \hat{F}\left(x_{(i)}\right)=\frac{i}{n+1} \quad \text { the mean rank) } \\
& \hat{F}\left(x_{(i)}\right)=\frac{i-0.5}{n} \\
& \hat{F}\left(x_{(i)}\right)=\frac{i-0.3}{n+0.4} \text { (the median rank) }
\end{aligned}
$$

where $i$ denotes the $i^{\text {th }}$ smallest value of $x_{(1)}, x_{(2)}, \ldots, x_{(n)}, i=1$, $2, \ldots, n$.

The estimates $\hat{\beta}_{0}$ and $\hat{\beta}_{1}$ of the regression parameters $\beta_{0}$ and $\beta_{1}$ minimize the function

$$
\begin{aligned}
& Q\left(\beta_{0}, \beta_{1}\right)=\sum_{i=1}^{n}\left(Y_{i}-\beta_{0}-\beta_{1} X_{i}\right)^{2}= \\
& =\sum_{i=1}^{n}\left(Y_{i}-\beta_{0}-\beta_{1} \ln x_{(i)}\right)^{2} .
\end{aligned}
$$

Therefore, the estimates $\hat{\beta}_{0}$ and $\hat{\beta}_{1}$ of the parameters $\beta_{0}$ and $\beta_{1}$ are given by

$$
\begin{gathered}
\hat{c}=\hat{\beta}_{1} \frac{n \sum_{i=1}^{n} \ln x_{(i)} \ln \left[-\ln \left(1-\hat{F}\left(x_{(i)}\right)\right)\right]-\sum_{i=1}^{n} \ln x_{(i)} \sum_{i=1}^{n} \ln \left[-\ln \left(1-\hat{F}\left(x_{(i)}\right)\right)\right]}{n \sum_{i=1}^{n} \ln ^{2} x_{(i)}-\left(\sum_{i=1}^{n} \ln x_{(i)}\right)}, \\
\hat{\beta}_{0}=\frac{1}{n} \sum_{i=1}^{n} \ln \left[-\ln \left(1-\hat{F}\left(x_{(i)}\right)\right)\right]-\hat{c} \frac{1}{n} \sum_{i=1}^{n} \ln x_{(i)} .
\end{gathered}
$$

The estimate $\hat{\delta}$ of the parameter $\delta$ is given by

$$
\hat{\delta}=\exp \left(-\frac{\hat{\beta}_{0}}{\hat{c}}\right)=\exp \left(-\frac{\sum_{i=1}^{n} \ln \left[-\ln \left(1-\hat{F}\left(x_{(i)}\right)\right)\right]-\hat{c} \sum_{i=1}^{n} \ln x_{(i)}}{\hat{c} n}\right) .
$$

\section{Weighted least square method}

We suppose that the estimates $\hat{\beta}_{0}$ and $\hat{\beta}_{1}$ of the regression parameters $\beta_{0}$ and $\beta_{1}$ minimize the function

$$
\begin{aligned}
& Q\left(\beta_{0}, \beta_{1}\right)=\sum_{i=1}^{n} w_{i}\left(Y_{i}-\beta_{0}-\beta_{1} X_{i}\right)^{2}= \\
& =\sum_{i=1}^{n} w_{i}\left(Y_{i}-\beta_{0}-\beta_{1} \ln x_{(i)}\right)^{2}
\end{aligned}
$$


where $w_{i}$ is the weight factor, $i=1,2, \ldots n$. Therefore, the esti-

To compare the performance of the various methods we mates $\hat{\beta}_{0}$ and $\hat{\beta}_{1}$ of the parameters $\beta_{0}$ and $\beta_{1}$ and are given by compute the sample root mean square error (RMSE) defined by

$$
\begin{aligned}
& \hat{c}=\hat{\beta}_{1}=\frac{\sum_{i=1}^{n} w_{i} \sum_{i=1}^{n} w_{i} \ln x_{(i)} \ln \left[-\ln \left(1-\hat{F}\left(x_{(i)}\right)\right)\right]-\sum_{i=1}^{n} w_{i} \ln x_{(i)} \sum_{i=1}^{n} w_{i} \ln \left[-\ln \left(1-\hat{F}\left(x_{(i)}\right)\right)\right]}{\sum_{i=1}^{n} w_{i} \sum_{i=1}^{n} w_{i} \ln ^{2} x_{(i)}-\left(\sum_{i=1}^{n} w_{i} \ln x_{(i)}\right)^{2}}, \\
& \hat{\beta}_{0}=\frac{\sum_{i=1}^{n} w_{i} \ln \left[-\ln \left(1-\hat{F}\left(x_{(i)}\right)\right)\right]-\hat{c} \sum_{i=1}^{n} w_{i} \ln x_{(i)}}{R M S E=\sqrt{\frac{1}{5000} \sum_{i=1}^{5000}\left[\left(\hat{c}_{i}-\bar{c}\right)^{2}+\left(\hat{\delta}_{i}-\bar{\delta}\right)^{2}\right]} .}
\end{aligned}
$$

$$
\hat{\beta}_{0}=\frac{\sum_{i=1}^{n} w_{i} \ln \left[-\ln \left(1-\hat{F}\left(x_{(i)}\right)\right)\right]-\hat{c} \sum_{i=1}^{n} w_{i} \ln x_{(i)}}{\sum_{i=1}^{n} w_{i}} .
$$

Then the estimate $\hat{\delta}$ of the parameter $\delta$ is given by

$$
\hat{\delta}=\exp \left(-\frac{\sum_{i=1}^{n} w_{i} \ln \left[-\ln \left(1-\hat{F}\left(x_{(i)}\right)\right)\right]-\hat{c} \sum_{i=1}^{n} w_{i} \ln x_{i}}{\hat{c} \sum_{i=1}^{n} w_{i}}\right) .
$$

In this paper we use the weight factor proposed by Bergman [1]

$$
w_{i}=\left[\left(1-\hat{F}\left(x_{(i)}\right)\right) \ln \left(1-\hat{F}\left(x_{(i)}\right)\right)\right]^{2}, i=1,2, \ldots, n .
$$

\section{Monte Carlo simulation}

We generate by the Monte Carlo simulation the random samples from the Weibull distribution and compare the performance of the methods for estimating the Weibull distribution parameters mentioned above. In simulation study we consider the LSM and the WLSM each with three methods for estimating the cumulative distribution function. Thus together we compare six methods. We denote the methods with the estimators of the cumulative distribution function (3) as LSM_1, WLSM_1, with (4) as LSM_2, WLSM_2 and with (5) as LSM_3, WLSM_3.

We consider sample sizes $n=5$ to $100, \delta=1$ and severa values of the parameters $c=0.5,1.5,2.5$ representing decreasing, increasing and concave, increasing and convex failure rate functions respectively. All possible combinations of the parameters $c$, $\delta$ and sample sizes $n$ are considered. For each combination $c, \delta$ and $n$ we generate by the Monte Carlo simulation $N=5000$ random samples from the Weibull distribution. For each of six methods we obtain 5000 estimates $\hat{c}_{1}, \hat{c}_{2}, \ldots, \hat{c}_{5000}$ of the parameter $c$ and 5000 estimates $\hat{\delta}_{1}, \hat{\delta}_{2}, \ldots, \hat{\delta}_{5000}$ of the parameter $\delta$. Then we compute for each method the sample means $\bar{c}, \bar{\delta}$ and the sample variances $s_{c}^{2}, s_{\delta}^{2}$, i. e.

$$
\begin{aligned}
& \bar{c}=\frac{1}{5000} \sum_{i=1}^{5000} \hat{c}_{i}, \bar{\delta}=\frac{1}{5000} \sum_{i=1}^{5000} \hat{\delta}_{i}, \\
& s_{c}^{2}=\frac{1}{4999} \sum_{i=1}^{5000}\left(\hat{c}_{i}-\bar{c}\right)^{2}, s_{\hat{\delta}}^{2}=\frac{1}{4999} \sum_{i=1}^{5000}\left(\hat{\delta}_{i}-\bar{\delta}\right)^{2} .
\end{aligned}
$$

The estimates with smaller variance and RMSE are prefered. The simulations and the calculation are performed in the Matlab system.

\section{Comparison of the methods}

In this section we summarize the performance of the methods for estimating the Weibull distribution parameters. The methods are compared in terms of the RMSE and sample size $n$.

The results of the comparison for selected sample sizes $n=5$, 10, 30, 50, 100 are summarized in Tables 1, 2, 3. The tables show the sample means, the sample variances and the sample RMSE. Figures 3, 4, 5 show illustrative plots of the $R M S E$ for $n=5$ to 50 (left), for $n=50$ to 100 (right).

It is evident that the RMSE of the least square method is in many cases much larger than the RMSE of the weighted least square method for the case studies in this paper. The weight factor improves the accuracy of the estimation the Weibull distribution parameters. When gets larger the RMSE of all methods tends to be smaller.

For the sample size $n \geq 10$ and for $c=1.5,2.5$ the comparison shows that the RMSE of the LSM_1 is in many cases much larger than the other methods. The LSM_2 and the LSM_3 are comparable methods in many cases in terms of the RMSE. The RMSE of the LSM_3 is slightly larger than the RMSE of the LSM_2. The RMSE of the WLSM_2 is larger than the WLSM_1 and the WLSM_3. The RMSE of the WLSM_3 is slightly larger than the RMSE of the WLSM_1, both methods are comparable for $n \geq 40$. In general, the WLSM_1 provides the best estimates of the Weibull distribution parameters than the other methods in terms of the RMSE.

For the small sample size $5 \leq n<10$ and for $c=1.5,2.5$ the comparison shows that in general the RMSE of the WLSM_1 outperforms the other methods. The RMSE of the LSM_1 is only slightly larger than the RMSE of the WLSM_1. The LSM_1 provides good results in these cases.

For the sample size $n \geq 10$ and for $c=0.5$ the comparison shows that in general the RMSE of the WLSM_2 outperforms the other methods. The RMSE of the WLSM_3 is slightly larger than the RMSE of the WLSM_2. The RMSE of the LSM_2 and the 
Simulation results of the parameter estimation for real parameters $c=0.5, \delta=1$

\begin{tabular}{|c|c|c|c|c|c|c|}
\hline $\begin{array}{c}\text { Sample } \\
\text { size }\end{array}$ & Method & $\bar{c}$ & $\bar{\delta}$ & $s_{c}^{2}$ & $s_{\delta}^{2}$ & $R M S E$ \\
\hline \multirow{6}{*}{$\mathrm{n}=5$} & LSM_1 & 0.4508 & 1.6745 & 0.0745 & 2.6689 & 1.7889 \\
\hline & LSM_2 & 0.6007 & 1.3947 & 0.1311 & 1.8255 & 1.4568 \\
\hline & LSM_3 & 0.5271 & 1.4977 & 0.1014 & 2.1039 & 1.5663 \\
\hline & WLSM_1 & 0.4319 & 1.6060 & 0.0651 & 2.4445 & 1.6974 \\
\hline & WLSM_2 & 0.5485 & 1.3710 & 0.1071 & 1.8767 & 1.4572 \\
\hline & WLSM_3 & 0.4940 & 1.4555 & 0.0855 & 2.0588 & 1.5334 \\
\hline \multirow{6}{*}{$\mathrm{n}=10$} & LSM_1 & 0.4363 & 1.3946 & 0.0205 & 0.9475 & 1.0619 \\
\hline & LSM_2 & 0.5311 & 1.2439 & 0.0298 & 0.7336 & 0.9075 \\
\hline & LSM_3 & 0.4860 & 1.3034 & 0.0252 & 0.8129 & 0.9645 \\
\hline & WLSM_1 & 0.4345 & 1.3256 & 0.0182 & 0.8595 & 0.9939 \\
\hline & WLSM_2 & 0.5036 & 1.2197 & 0.0272 & 0.7586 & 0.9132 \\
\hline & WLSM_3 & 0.4734 & 1.2595 & 0.0226 & 0.7937 & 0.9403 \\
\hline \multirow{6}{*}{$\mathrm{n}=30$} & & & & & & 0.5108 \\
\hline & LSM_2 & 0.5048 & 1.0972 & 0.0090 & 0.1957 & 0.4627 \\
\hline & LSM_3 & 0.4822 & 1.1246 & 0.0084 & 0.2078 & 0.4816 \\
\hline & WLSM_1 & 0.4713 & 1.1046 & 0.0066 & 0.1984 & 0.4655 \\
\hline & WLSM_2 & 0.4966 & 1.0721 & 0.0079 & 0.1898 & 0.4503 \\
\hline & WLSM_3 & 0.4864 & 1.0847 & 0.0073 & 0.1930 & 0.4557 \\
\hline \multirow{6}{*}{$\mathrm{n}=50$} & LSM_1 & 0.4652 & 1.1087 & 0.0047 & 0.1198 & 0.3708 \\
\hline & LSM_2 & 0.5011 & 1.0637 & 0.0053 & 0.1083 & 0.3430 \\
\hline & LSM_3 & 0.4848 & 1.0831 & 0.0051 & 0.1131 & 0.3540 \\
\hline & WLSM_1 & 0.4823 & 1.0640 & 0.0040 & 0.1108 & 0.3452 \\
\hline & WLSM_2 & 0.4976 & 1.0450 & 0.0045 & 0.1080 & 0.3383 \\
\hline & WLSM_3 & 0.4915 & 1.0524 & 0.0043 & 0.1091 & 0.3408 \\
\hline \multirow{6}{*}{$\mathrm{n}=100$} & LSM_1 & 0.4760 & 1.0563 & 0.0025 & 0.0533 & 0.2440 \\
\hline & LSM_2 & 0.4991 & 1.0283 & 0.0027 & 0.0501 & 0.2315 \\
\hline & LSM_3 & 0.4888 & 1.0405 & 0.0026 & 0.0515 & 0.2363 \\
\hline & WLSM_1 & 0.4898 & 1.0258 & 0.0022 & 0.0525 & 0.2355 \\
\hline & WLSM_2 & 0.4974 & 1.0166 & 0.0023 & 0.0519 & 0.2333 \\
\hline & WLSM_3 & 0.4944 & 1.0202 & 0.0023 & 0.0521 & 0.2341 \\
\hline
\end{tabular}

Simulation results of the parameter estimation for real parameters $c=1.5, \delta=1$

\begin{tabular}{|c|c|c|c|c|c|c|}
\hline $\begin{array}{c}\text { Sample } \\
\text { size }\end{array}$ & Method & $\bar{c}$ & $\bar{\delta}$ & $s_{c}^{2}$ & $s_{\delta}^{2}$ & $R M S E$ \\
\hline \multirow{5}{*}{$\mathrm{n}=5$} & LSM_1 & 1.3407 & 1.0925 & 0.7156 & 0.1192 & 0.9320 \\
& LSM_2 & 1.7878 & 1.0277 & 1.2710 & 0.1060 & 1.2084 \\
& LSM_3 & 1.5683 & 1.0527 & 0.9784 & 0.1107 & 1.0471 \\
& WLSM_1 & 1.2914 & 1.0764 & 0.6761 & 0.1170 & 0.9178 \\
& WLSM_2 & 1.6453 & 1.0164 & 1.1606 & 0.1095 & 1.1363 \\
& WLSM_3 & 1.4792 & 1.0394 & 0.9075 & 0.1117 & 1.0105 \\
\hline \multirow{5}{*}{$\mathrm{n}=10$} & LSM_1 & 1.2949 & 1.0598 & 0.1758 & 0.0564 & 0.5270 \\
& LSM_2 & 1.5759 & 1.0202 & 0.2545 & 0.0519 & 0.5590 \\
& LSM_3 & 1.4421 & 1.0363 & 0.2154 & 0.0536 & 0.5232 \\
& WLSM_1 & 1.2884 & 1.0413 & 0.1562 & 0.0554 & 0.5079 \\
& WLSM_2 & 1.4920 & 1.0108 & 0.2348 & 0.0542 & 0.5378 \\
& WLSM_3 & 1.4033 & 1.0226 & 0.1948 & 0.0545 & 0.5091 \\
\hline \multicolumn{7}{|c|}{}
\end{tabular}

\begin{tabular}{|c|c|c|c|c|c|c|}
\hline \multirow{5}{*}{$\mathrm{n}=30$} & LSM_1 & 1.3653 & 1.0326 & 0.0676 & 0.0185 & 0.3245 \\
& LSM_2 & 1.5126 & 1.0135 & 0.0801 & 0.0176 & 0.3131 \\
& LSM_3 & 1.4447 & 1.0217 & 0.0743 & 0.0180 & 0.3095 \\
& WLSM_1 & 1.4098 & 1.0162 & 0.0569 & 0.0181 & 0.2888 \\
& WLSM_2 & 1.4853 & 1.0059 & 0.0677 & 0.0181 & 0.2932 \\
& WLSM_3 & 1.4549 & 1.0099 & 0.0630 & 0.0181 & 0.2885 \\
\hline \multirow{5}{*}{$\mathrm{n}=50$ 0 } & & & & & \\
& LSM_1 & 1.3945 & 1.0218 & 0.0433 & 0.0112 & 0.2570 \\
& LSM_2 & 1.5022 & 1.0080 & 0.0485 & 0.0107 & 0.2435 \\
& LSM_3 & 1.4532 & 1.0140 & 0.0462 & 0.0109 & 0.2438 \\
& WLSM_1 & 1.4442 & 1.0083 & 0.0369 & 0.0110 & 0.2261 \\
& WLSM_2 & 1.4897 & 1.0022 & 0.0412 & 0.0110 & 0.2286 \\
& WLSM_3 & 1.4716 & 1.0046 & 0.0394 & 0.0110 & 0.2264 \\
& LSM_1 & 1.4276 & 1.0155 & 0.0240 & 0.0054 & 0.1868 \\
& LSM_2 & 1.4968 & 1.0065 & 0.0255 & 0.0053 & 0.1755 \\
& LSM_3 & 1.4657 & 1.0104 & 0.0248 & 0.0053 & 0.1774 \\
& WLSM_1 & 1.4711 & 1.0052 & 0.0200 & 0.0054 & 0.1619 \\
& WLSM_2 & 1.4938 & 1.0022 & 0.0211 & 0.0054 & 0.1628 \\
& WLSM_3 & 1.4847 & 1.0034 & 0.0206 & 0.0054 & 0.1620 \\
\hline
\end{tabular}

Simulation results of the parameter estimation

Table 1 for real parameters $c=2.5, \delta=1$

\begin{tabular}{|c|c|c|c|c|c|c|}
\hline $\begin{array}{c}\text { Sample } \\
\text { size }\end{array}$ & Method & $\bar{c}$ & $\bar{\delta}$ & $s_{c}^{2}$ & $s_{\delta}^{2}$ & $R M S E$ \\
\hline \multirow{6}{*}{$n=5$} & LSM_1 & 2.2223 & 1.0423 & 1.5070 & 0.0387 & 1.2745 \\
\hline & LSM_2 & 2.9630 & 1.0048 & 2.6661 & 0.0361 & 1.7076 \\
\hline & LSM_3 & 2.5993 & 1.0194 & 2.0564 & 0.0370 & 1.4503 \\
\hline & WLSM_1 & 2.1367 & 1.0329 & 1.3909 & 0.0383 & 1.2498 \\
\hline & WLSM_2 & 2.7187 & 0.9975 & 2.4000 & 0.0377 & 1.5764 \\
\hline & WLSM_3 & 2.4459 & 1.0112 & 1.8685 & 0.0377 & 1.3816 \\
\hline \multirow{6}{*}{$\mathrm{n}=10$} & LSM_1 & 2.1727 & 1.0325 & 0.5197 & 0.0197 & 0.8047 \\
\hline & LSM_2 & 2.6437 & 1.0094 & 0.7517 & 0.0188 & 0.8894 \\
\hline & LSM_3 & 2.4194 & 1.0188 & 0.6367 & 0.0191 & 0.8140 \\
\hline & WLSM_1 & 2.1559 & 1.0212 & 0.4450 & 0.0196 & 0.7637 \\
\hline & WLSM_2 & 2.4961 & 1.0028 & 0.6570 & 0.0196 & 0.8225 \\
\hline & WLSM_3 & 2.3478 & 1.0100 & 0.5497 & 0.0195 & 0.7696 \\
\hline \multirow{6}{*}{$\mathrm{n}=30$} & LSM_1 & 2.2736 & 1.0151 & 0.1894 & 0.0064 & 0.4973 \\
\hline & LSM_2 & 2.5190 & 1.0038 & 0.2245 & 0.0062 & 0.4807 \\
\hline & LSM_3 & 2.4060 & 1.0086 & 0.2082 & 0.0063 & 0.4727 \\
\hline & WLSM_1 & 2.3494 & 1.0056 & 0.1656 & 0.0064 & 0.4412 \\
\hline & WLSM_2 & 2.4747 & 0.9994 & 0.1977 & 0.0064 & 0.4525 \\
\hline & WLSM_3 & 2.4243 & 1.0018 & 0.1839 & 0.0064 & 0.4427 \\
\hline \multirow{6}{*}{$\mathrm{n}=50$} & LSM_1 & 2.3228 & 1.0145 & 0.1193 & 0.0040 & 0.3935 \\
\hline & LSM_2 & 2.5024 & 1.0062 & 0.1332 & 0.0039 & 0.3703 \\
\hline & LSM_3 & 2.4206 & 1.0098 & 0.1269 & 0.0039 & 0.3704 \\
\hline & WLSM_1 & 2.4116 & 1.0065 & 0.0997 & 0.0039 & 0.3339 \\
\hline & WLSM_2 & 2.4876 & 1.0029 & 0.1112 & 0.0040 & 0.3396 \\
\hline & WLSM_3 & 2.4573 & 1.0043 & 0.1065 & 0.0040 & 0.3350 \\
\hline \multirow{6}{*}{$\mathrm{n}=100$} & LSM_1 & 2.3805 & 1.0082 & 0.0646 & 0.0019 & 0.2844 \\
\hline & LSM_2 & 2.4960 & 1.0028 & 0.0686 & 0.0019 & 0.2656 \\
\hline & LSM_3 & 2.4441 & 1.0052 & 0.0669 & 0.0019 & 0.2682 \\
\hline & WLSM_1 & 2.4580 & 1.0021 & 0.0539 & 0.0019 & 0.2399 \\
\hline & WLSM_2 & 2.4959 & 1.0003 & 0.0568 & 0.0019 & 0.2424 \\
\hline & WLSM_3 & 2.4808 & 1.0010 & 0.0556 & 0.0019 & 0.2407 \\
\hline
\end{tabular}



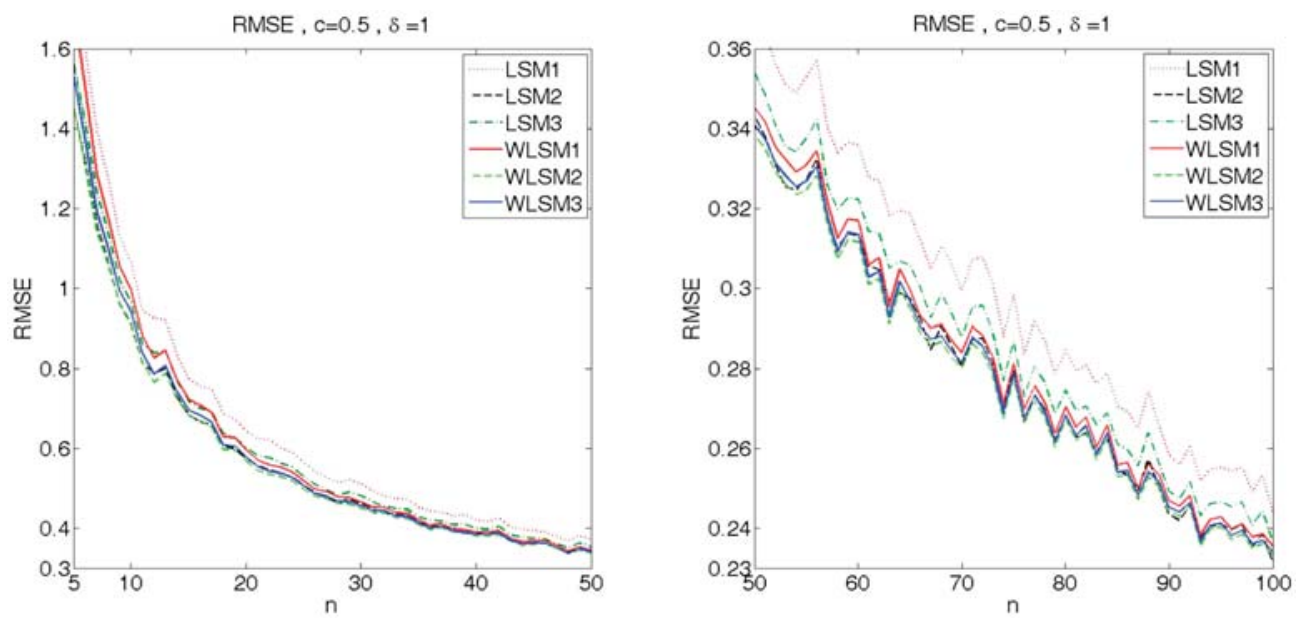

Fig. 3 Root mean square error for real parameters $c=0.5, \delta=1$
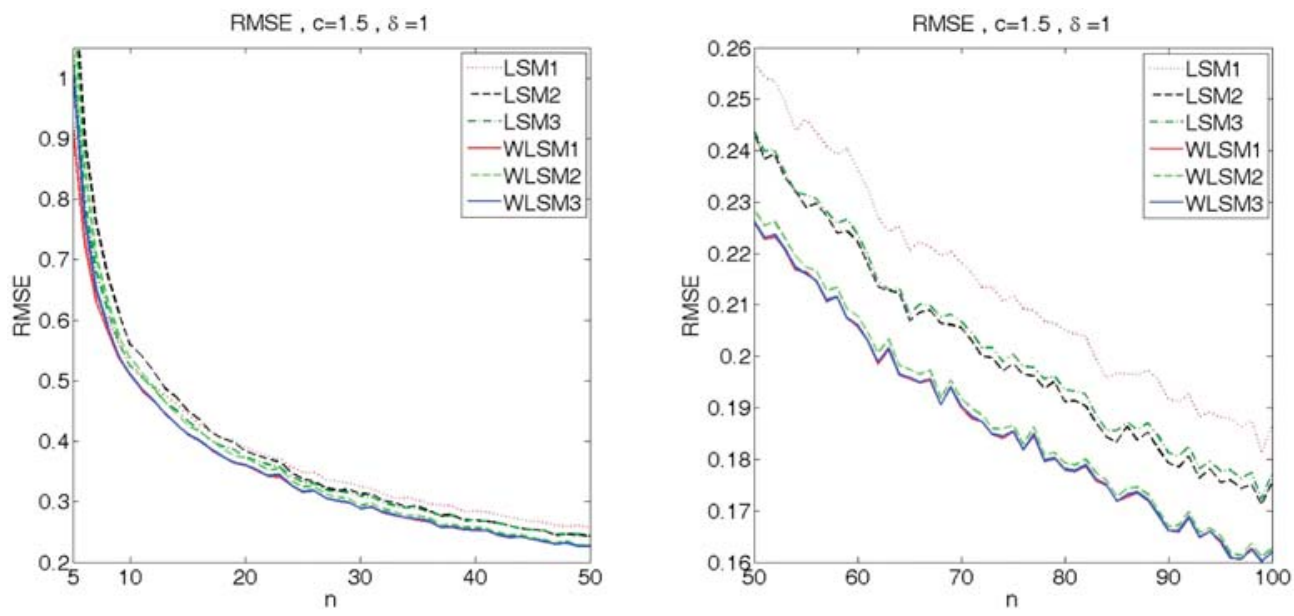

Fig. 4 Root mean square error for real parameters $c=1.5, \delta=1$
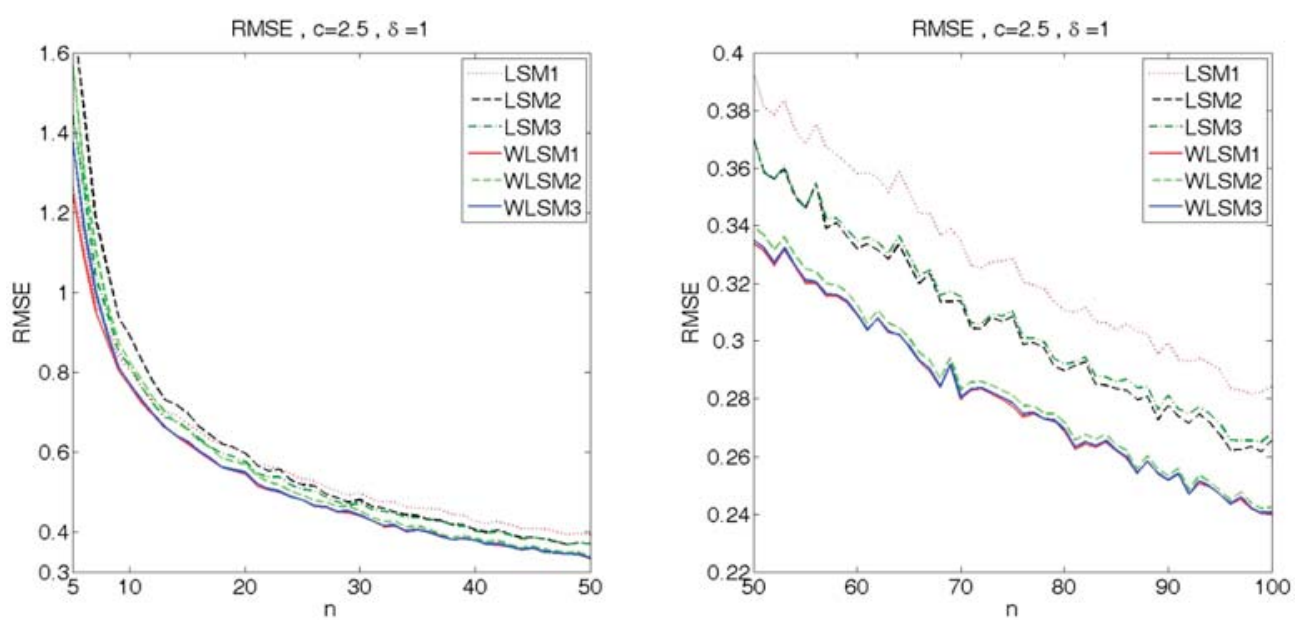

Fig. 5 Root mean square error for real parameters $c=2.5, \delta=1$ 
WLSM_1 follow. The RMSE of the LSM_1 is in many cases much larger than the other methods.

For the sample size $5 \leq n<10$ and for $c=0.5$ the RMSE of the LSM_2 is only slightly larger than the RMSE of the WLSM_2. The RMSE of the WLSM_3 and the LSM_3 follow. The RMSE of the LSM_1 is much larger than the RMSE of the other methods.

\section{Concluding remarks}

In this paper we compared the performance of the methods for estimating the Weibull distribution parameters in terms of the $R M S E$ and sample size $n$. The comparison was based on the Monte Carlo simulation. The comparison shows that the weight factor improves the accuracy of the estimation the Weibull distribution parameters. The WLSM_1 performs the best in terms of the RMSE than the other methods for majority cases studied in this paper and for all sample sizes, the WLSM_3 follows as the second good choice. Except the case when $c=0.5$, the WLSM_2 performs to be the best for all the sample sizes than the other methods. The good choice is in this case for middle and large sample sizes the WLSM_3.

The advantages of these recommended methods are: simple derivation, easy calculation of the estimates of the parameters by the closed-form formula. And so from this point of view these methods are very useful for engineering practice.

\section{Acknowledgement}

This research was supported by the Slovak Grant Agency VEGA through the projects No. 1/1245/12 and No. 1/0797/12.

\section{References}

[1] BERGMAN, B.: Estimation of Weibull Parameters Using a Weight Function. J. of Materials Science Letters 5, pp. 611-614, 1986

[2] CHU, Y. K., KE, CH., J.: Computation Approaches for Parameter Estimation of Weibull Distribution. Mathematical and Computational Applications, vol. 17, No. 1, pp. 39-47, 2012

[3] FAUCHER, B., TYSON, W. R.: On the Determination of Weibull Parameters. J. of Materials Science Letters 7, pp. 1199-1203, 1988

[4] LU, H. L., CHEN, CH. H., WU, J. W.: A Note on Weighted Least-squares Estimation of the Shape Parameter of the the Weibull Distribution. Quality and Reliability Engineering International 20, pp. 579-586, 2004

[5] TRUSTRUM, K., JAYATILAKA, A. S.: On Estimating the Weibull Modulus for a Brittle Material. J. of Material Science 14, pp. $1080-1084,1979$

[6] WU, D., ZHOU, J., LI, Y.: Methods for Estimating Weibull Parameters for Brittle Materials. J. of Material Science 41, pp. 5630-5638, 2006

[7] ZERDA, I.: An Experimental Comparison of Popular Estimation Methods for the Weibull, Gamma, and Gompertz Distributions. Schedae Informaticae 20, pp. 67-82, 2011. 